\title{
Ultrasonic rangefinder with resolution in hundredths of the probing signal's wavelength for the mobile rescue robot
}

\author{
Stanislaw Goll ${ }^{1,2}$, Julia Maximova ${ }^{1,2}$ \\ ${ }^{1}$ Department of Information-Measuring and Biomedical Engineering, Ryazan State Radio Engineering University (RSREU), Gagarin Street \\ 59/1, Ryazan, Russia \\ ${ }^{2}$ LLC KB Avrora, Skomoroshinskaja Street 9, of.3, Ryazan, Russia
}

\section{ABSTRACT}

The main goal of this research is to increase the measurement resolution of ultrasonic rangefinders to meet the needs of vital signs noncontact registration based on chest movements. The two-phase method is proposed to make distance estimates by sending probe pulse trains, calculating the phase spectrum of the echo signal's envelope, and tracking its relevant components. During the first phase, rough Time-of-Flight (ToF)-based estimates are made. During the second phase, this estimate is corrected based on the phase spectrum of the echo signal's envelope, the phase ambiguity is removed, and the relevant components are determined. The final estimate of the human chest displacement is calculated based on these relevant components. The output data rate is the same as for the ToF-based measurements, but the measurement resolution is increased to one hundredth of the ultrasonic wavelength. The experiment results are provided for the both model and the real human chest displacements caused by the respiration and heartbeat processes.

\section{Section: RESEARCH PAPER}

Keywords: ultrasound; rangefinder; resolution; envelope; phase spectrum; rescue robot

Citation: Stanislaw Goll, Julia Maximova, Ultrasonic rangefinder with resolution in hundredths of the probing signal's wavelength for the mobile rescue robot, Acta IMEKO, vol. 8, no. 4, article 8, December 2019, identifier: IMEKO-ACTA-08 (2019)-04-08

Editor: Yvan Baudoin, International CBRNE Institute, Belgium

Received November 23, 2018; In final form June 26, 2019; Published December 2019

Copyright: This is an open-access article distributed under the terms of the Creative Commons Attribution 3.0 License, which permits unrestricted use, distribution, and reproduction in any medium, provided the original author and source are credited.

Corresponding author: Julia Maximova, e-mail: maximova@kb-avrora.ru

\section{INTRODUCTION}

Extreme mobile robotic systems, safety-critical systems (particularly those designed for drivers, pilots, and motormen), and medical equipment (e.g. diagnostic and therapy instruments) need low-cost precision rangefinders with an effective range of up to $1 \mathrm{~m}$ and an output data rate of at least $20 \mathrm{~Hz}$ to measure both absolute and relative distances. Such rangefinder sensors can be used to provide the noncontact measurements of a person's pulse and respiratory rates (Figure 1) - information that is critical for planning rescue operations, the prevention of human-factor accidents, and the synchronisation of medical equipment with a patient's biorhythms [1].

Search and Rescue (SAR) operations caused by industrial disasters and military conflicts can be dangerous for SAR crew members. The main need here is to minimise the human presence in the danger zone by relying on telemetry data (i.e. victims' vital signs and locations) to optimally plan and execute the rescue operation, including the SAR crew members' efforts and the rescue robots, both autonomous and remote controlled [2], [3].
Detection of the injured person's vital signs, mainly pulse and respiratory rates, poses a nontrivial task even for a remote controlled rescue robot [4], [6]. For that task, video and thermal cameras are considered to be the most informative sensors. Due to the amount of data broadcasted by these sensors, they require a wired connection (e.g. a LAN) between the robot and the human operator or the broadband wireless connection in the case that the former option is not possible. However, the radio wave-based data transmission can lead to detection mistakes due

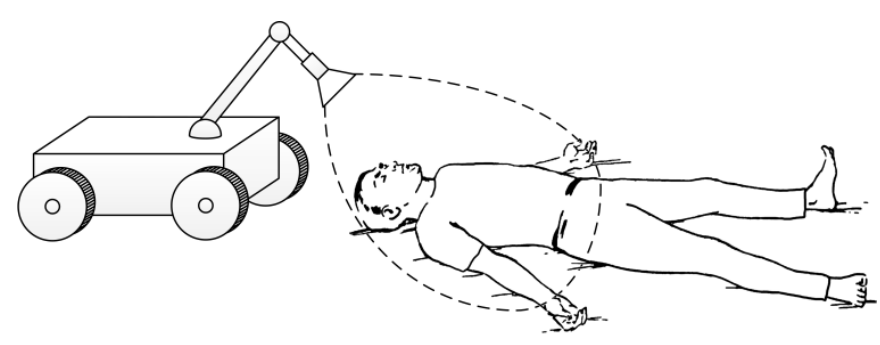

Figure 1. Positioning of the ultrasonic beam pattern by the mobile robot in noncontact registration of the person's pulse and respiratory rates. 
to interferences and non-line-of-sight and near-line-of-sight transmissions. Moreover, the usage of thermal cameras to detect body temperature can sometimes lead to a dead body being mistaken for a living person.

Another way of measuring human vital signs is to make contact measurements [7]. In that case, the robot must place electrodes on particular parts of the human body, which leads to many problems, like the process of electrode placement or the detection and recognition of the body parts where the electrodes should be placed. Clearly, these can be quite dangerous operations to be performed on an injured person.

In section 2, we will discuss the relevant prior research in this area, including the advantages and disadvantages thereof. In the next section, the essence of the proposed method is described. The following section presents some experiments and their results. In the final section, conclusions are made and recommendations for further research are outlined.

\section{RELATED WORK}

The noncontact measurements of the pulse and respiratory rates can be taken by means of short-range radars [8], [10], since it is possible for a single integrated circuit to contain two $77 \mathrm{GHz}$ UHF transmission lines (one transmitting line and one receiving line) as well as the low-frequency processing channel and the ADC [11]. The data gathered that way can be affected by electromagnetic interference and other factors; hence, we propose to increase the robustness of the radar measurements by means of the secondary noncontact measurement channel based on ultrasonic sensors.

The commonly used Time-of-Flight (ToF) principle [12], [13], is based on measurement of the time interval between the start of the ultrasonic wave emission and the moment when the reflected wave causes the difference between the current air pressure and the air pressure at rest to exceed the given threshold for the first time. The measurement resolution of the ToF-based sensor depends both on the properties of the timer/counter used and the ultrasonic signal wavelength. The ToF measurement resolution for the $40 \mathrm{kHz}$ ultrasound is, at a rough estimate, 8 $\mathrm{mm}$. At the same time, many of the ToF-based sensors are featured, with the resolution being up to one fourth of the ultrasonic wavelength. To achieve such resolution, various methods can be used: the adaptive threshold, comparisons for the positive and negative half-waves, etc. However, all these modifications are not enough to achieve the submillimetre resolution.

Further attempts to improve the measurement resolution of the ultrasonic rangefinders are based on linear frequency modulation or composite modulation [14]; binary frequency shift-keyed signal and phase detection [15]; Vernier caliper phase metre [16]; and so on. However, these methods require broadband ultrasonic receivers and transmitters, sufficiently increasing the hardware cost.

\section{THE APPROACH}

In this article, we propose an algorithm to improve the measurement resolution of the low-cost single-tone $40 \mathrm{kHz}$ ultrasonic rangefinders. These on the inverse piezoelectric effect based sensors are commonly used in the automotive industry to build Advanced Driver Assistance Systems (ADASs) [17].

The cyclic movements of the human chest are caused by both the respiration process and cardiac activity, with movement amplitudes of 4 to $12 \mathrm{~mm}$ and $0.5 \mathrm{~mm}$ accordingly [18]. The cardiac cycle is more frequent than the respiration cycle and can occur up to 200 times per minute. According to the NyquistShannon-Kotelnikov sampling theorem, the measurement rate of $20 \mathrm{~Hz}$ is sufficient to perform vital signs detection. Due to the comparatively high sound speed in the air medium, it is possible to obtain up to 100 measurements per second at a distance of up to $1 \mathrm{~m}$ using the ToF method. Nevertheless, the measurement resolution of one fourth of the $40 \mathrm{kHz}$ ultrasonic wavelength is deficient in pulse and respiratory rates registration.

We suggest an approach to improve the measurement resolution without changing the output data rate. First, some method (for example, the ToF method) is used to obtain a rough

a

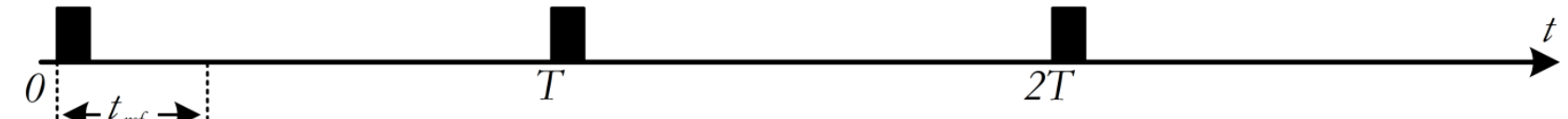

$\mathrm{b}$

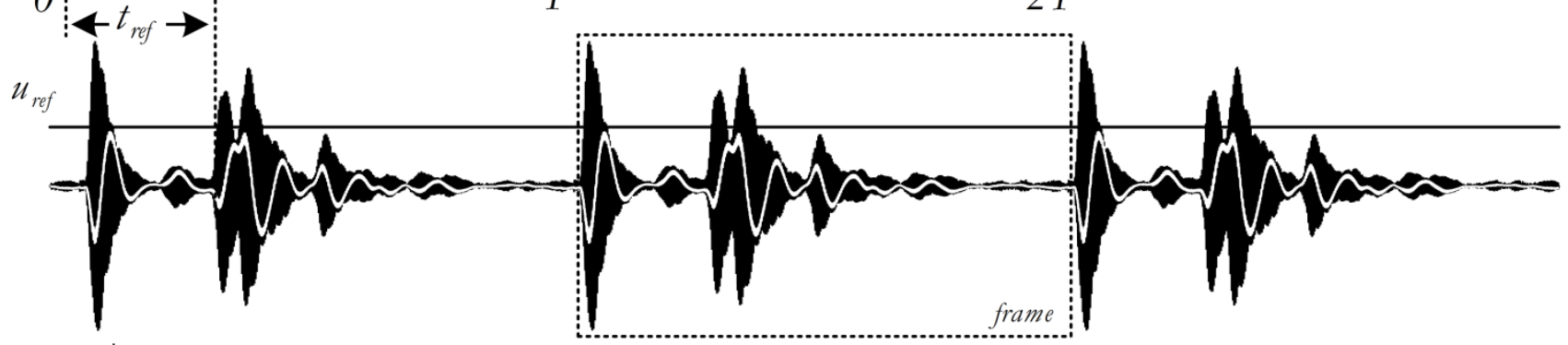

C

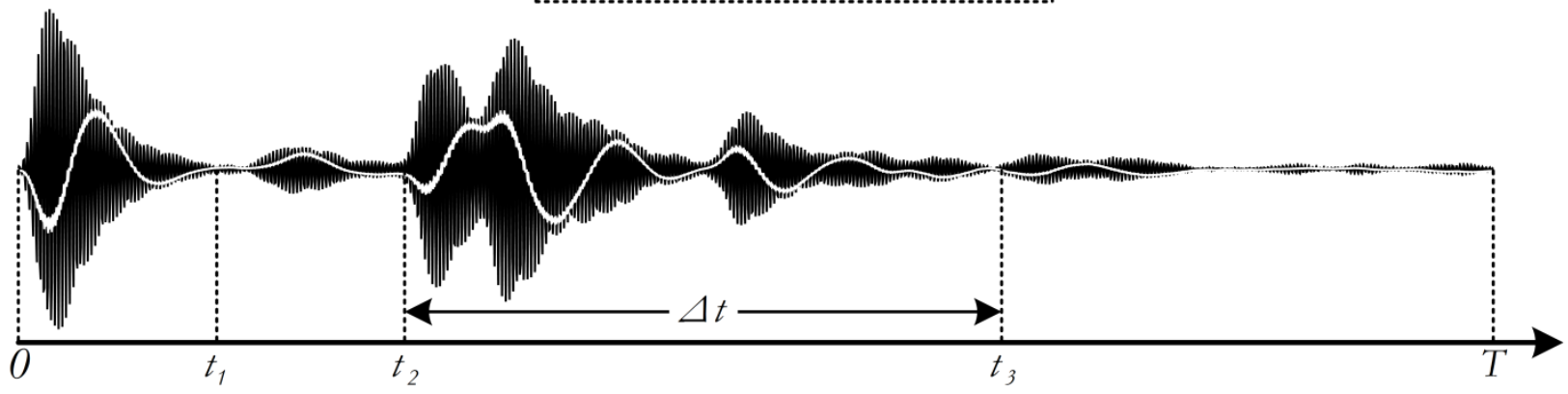

Figure 2. (a) Probe pulse trains (b) Received reflected signal (c) A single frame as it appears in the receiver. 
distance estimate $d_{r e f}$. Second, the proposed method is used to estimate the displacement $d$ relative to the rough estimate $d_{r e f}$

. Contrary to the ToF method, which uses the point in time at which the threshold is exceeded as the input data, the proposed method uses all the readings of the echo signal gathered with the sampling rate $f_{d}=200 \mathrm{kHz}$.

Figure 2(a) shows pulse trains containing $N=8$ probe pulses each with the probe pulse frequency set to $f_{u s}=40 \mathrm{kHz}$. The pulse trains are transferred to ultrasonic transmitter with the frame period being $T=10 \mathrm{~ms}$. The black-coloured regions in Figure 2(b) represent the digital signal $u$, which is formed from the received frame sequence and can be described as the amplitude overmodulated signal. The white line in Figure 2(b) represents the demodulated envelope of the receiver's signal. To compute the envelope, the modulated signal is multiplied by the sinusoidal carrier signal $v=V \sin \left(2 \pi f_{u s} t+\varphi_{v}\right)$ sampled with rate $f_{d}$; the low pass recursive filter with the transfer function $\frac{b_{0} z^{2}+b_{1} z+b_{2}}{z^{2}+a_{1} z+a_{2}}$ is then applied to the multiplication result. Figure 2(c) shows an enlarged version of the frame bordered by the dotted rectangle in Figure 2(b). Each frame contains the deterministic transmitted pulse received when the transmitter emits the ultrasonic wave. This signal starts from the beginning of the frame and ends at time $t_{1}$. In the case that some object or group of objects is present in the area covered by the rangefinder's beam pattern and these objects are large enough to reflect the ultrasonic wave with the frequency $f_{u s}$, the frames also contain the reflected echo signal at the time interval $\Delta t=\left[t_{2}, t_{3}\right]$.

Moment $t_{1}$ is when the transmitted pulse ends is constant for every frame and is computed as $t_{1}=\frac{N+n_{1}}{f_{u s}}$. We define the frame containing the rough ToF-based measurement of the distance $d_{r e f}$ to the closest object in the sensor's beam pattern as a reference frame. The rough estimate $d_{r e f}$ is proportional to the time interval $t_{r f}$, which is determined when the echo signal exceeds the comparison threshold $u_{r e f}$ after time $t_{1}$. For every frame received after the reference frame time $t_{2}=t_{r f}-\frac{n_{2}}{f_{u s}}$ remains constant until the new reference frames arrive, changing the value of $t_{r e f}$. The coefficients $n_{1}$ and $n_{2}$ represent the adjustable offsets with the integer values.

The reference frame is used as a starting point for the proposed method to compute its distance estimate and can be set (and changed later) either by the human operator's request or automatically if the absolute distance measurements acquired by means of the ToF method lie in some predetermined intervals. The moment $t_{3}=t_{2}+\Delta t$ sets the right boundary of the echo signal's time interval of the constant duration $\Delta t=$ const .

On the $\Delta t=\left[t_{2}, t_{3}\right]$ interval of each frame, Fast Fourier Transform is applied to the echo signal's envelope, and then, for each of its frames, the phase spectrum is computed.
The next step is the selection of the $M=10$ sequential spectrum components (starting with the second component), which are expressed as $\vec{\varphi}[k]=\left(\begin{array}{llll}\varphi_{1}[k] & \varphi_{2}[k] & \ldots & \varphi_{M}[k]\end{array}\right)^{T}$, where $k$ is the number of frames relative to the reference frame. This selection takes place at the end of each frame period $T$. Each component $\varphi_{m}[k]$ is used to estimate the displacement $d_{m}[k]$ relative to the rough estimate $d_{r f}$. This estimate is calculated as $d_{m}[k]=\beta \cdot \Phi_{m}[k]$, where $\beta$ is the conversion coefficient $(\mathrm{m} / \mathrm{rad}) ; \Phi_{m}[k]=\varphi_{m}[k]+\lambda_{m}[k] \cdot 2 \pi$ is the absolute value of the phase spectrum $m^{\text {th }}$ component after unwrapping; and $\lambda_{m}[k]$ is the number of $m^{\text {th }}$ component phase unwrappings and is calculated as follows:

$$
\lambda_{m}[k]=\lambda_{m}[k-1]+g\left(\Delta \varphi_{m}[k]\right) \text {, }
$$

where $\Delta \varphi_{m}[k]=\varphi_{m}[k]-\varphi_{m}[k-1]$,

$g(\xi)=\left\{\begin{array}{l}-1, \xi<-\varphi_{r f}, \\ 1, \xi>\varphi_{r f}, \\ 0,-\varphi_{r f} \leq \xi \leq \varphi_{r f},\end{array}\right.$

$\varphi_{\text {ref }}$ is the adjustable threshold of the $\Delta \varphi_{m}[k]$.

Figure 3 illustrates the computation of $\Phi_{m}[k]$. When the new reference frame is set, the number of phase unwrappings $\lambda_{m}[0]$ is zeroed for each phase component, which provides the recurrent Equation (1) with the initial condition. Therefore, for each received frame, $M=10$ estimates are calculated for the absolute distance $h_{m}$ between the sensor and the object of interest. Each of these estimates is calculated as $b_{m}[k]=d_{r f}+d_{m}[k]$, where $m=1,2, \ldots, M$ and apparently

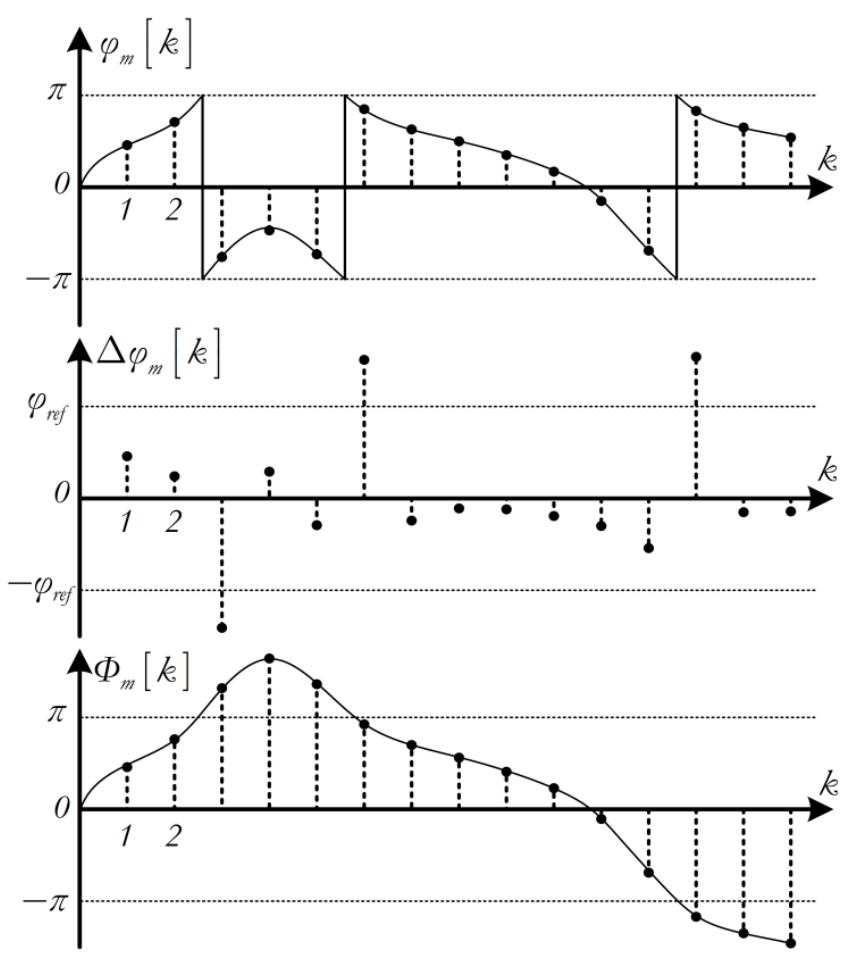

Figure 3. Phase unwrapping. 
Algorithm: displacement estimation

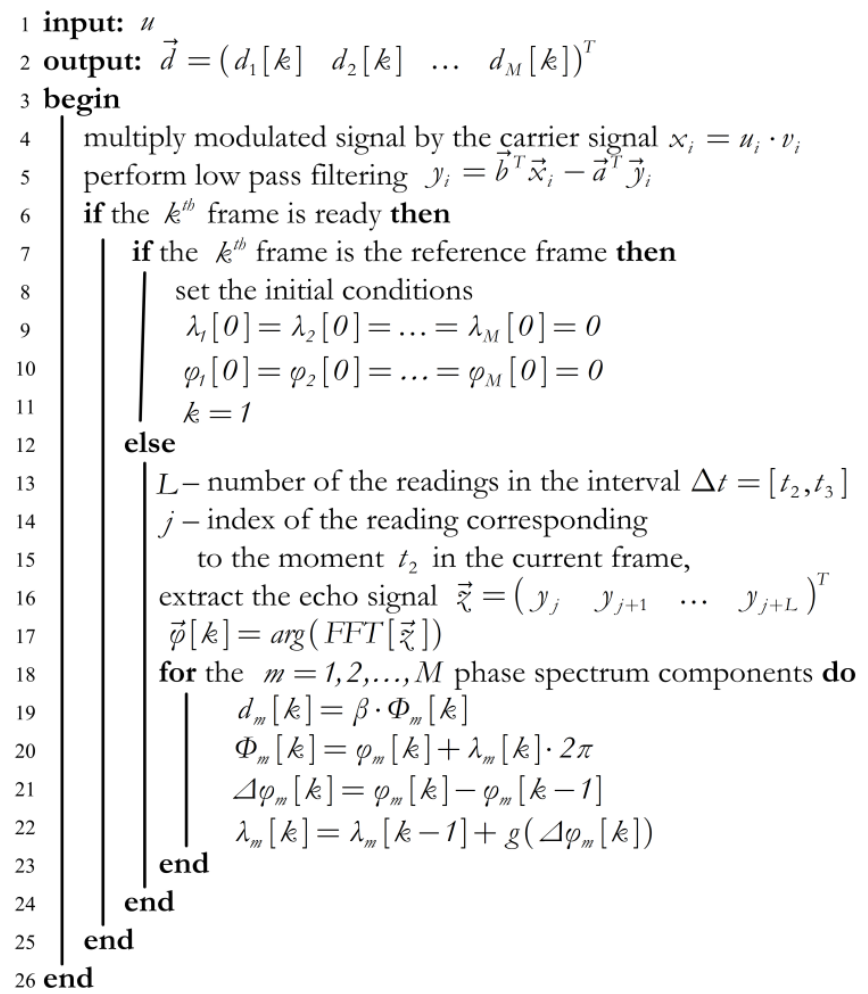

represents the sum of the reference distance $d_{r e f}$ and the displacement $d_{m}[k]$ computed using the proposed method. Let us note that $N, n_{1}, n_{2}, u_{r e f}, \Delta t, \varphi_{r f}, \vec{a}=\left[\begin{array}{ll}a_{1} & a_{2}\end{array}\right]^{T}$,
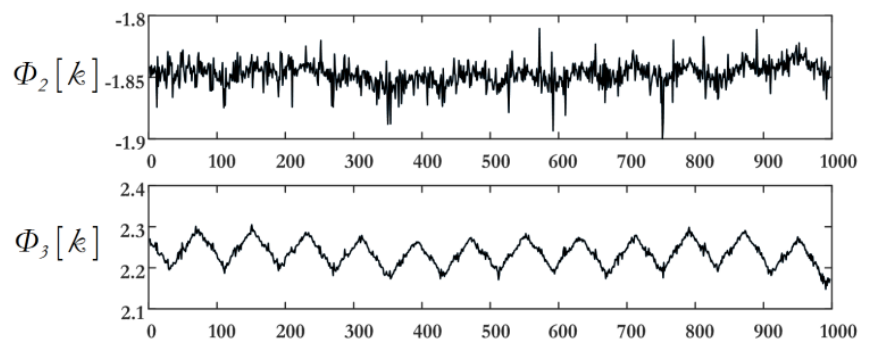

a
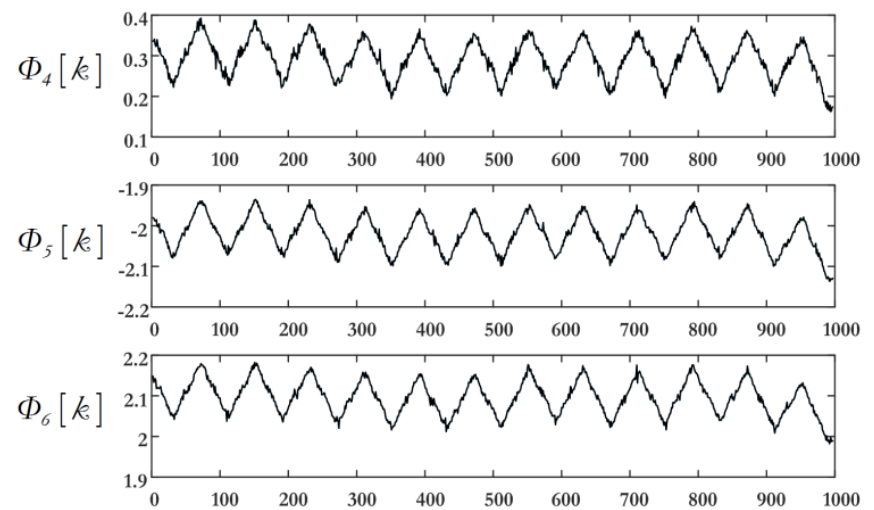

$\mathrm{b}$

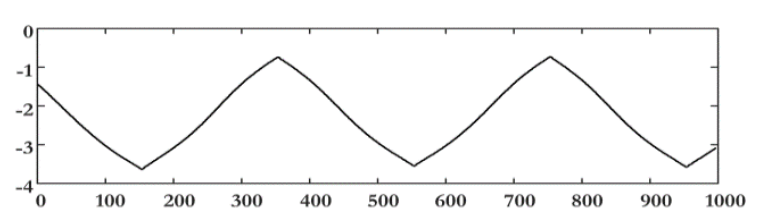

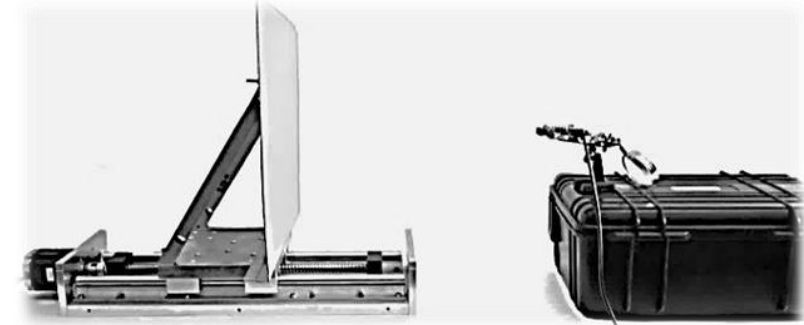

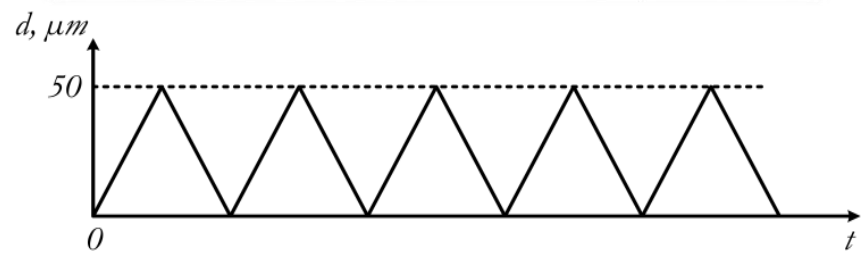

Figure 4. The experimental setup and the sawtooth-like displacements of the sheet.

$\vec{b}=\left[\begin{array}{lll}b_{1} & b_{2} & b_{3}\end{array}\right]^{T}, M$ are the structural parameters of the algorithm and are estimated empirically before the algorithm is applied.

\section{RESULTS}

The proposed method was used to process data gathered from the ultrasonic rangefinder in the three experiments. For the first experiment, a rectangular aluminium sheet was used as the object of interest, shown in Figure 4. A step motor with a step of $1.56 \mu \mathrm{m}$ was used to cause the periodic sawtooth-like motion of the sheet with an amplitude of $50 \mu \mathrm{m}$ along the axis of the rangefinder's beam pattern.
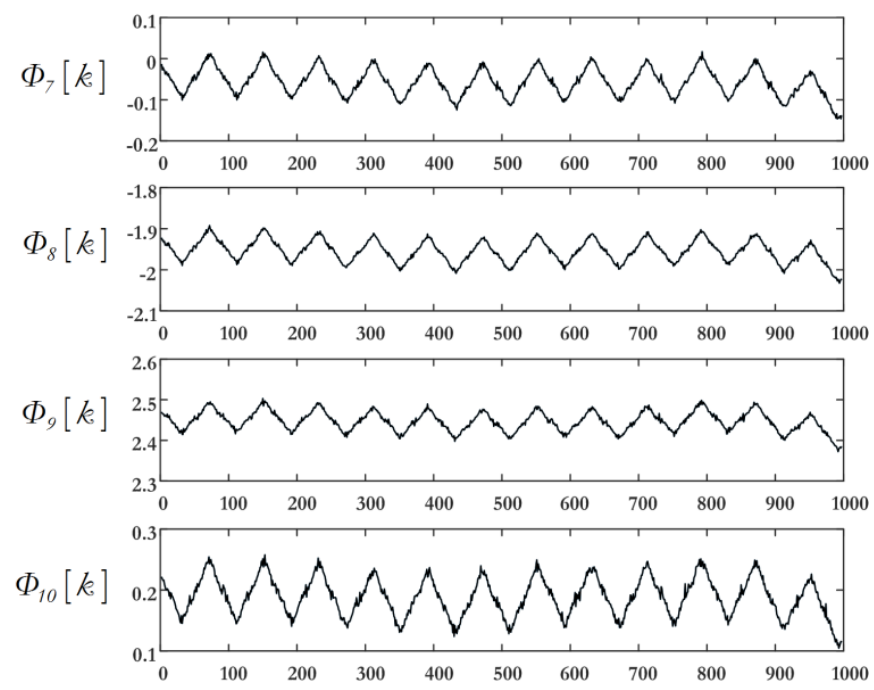

$\Phi_{11}[k]$
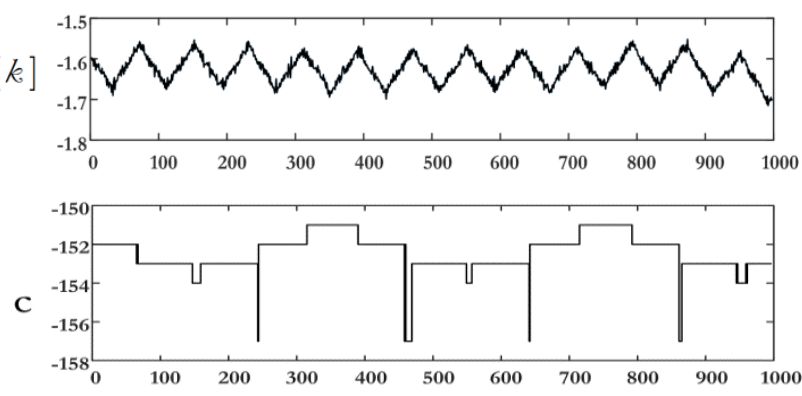

Figure 5. Changes in absolute values of the phase spectrum components $\Phi_{m}[k]$ caused by aluminium sheet displacement (proposed method): a - amplitude of $50 \mu \mathrm{m}, \mathrm{b}$-amplitude of $4 \mathrm{~mm}$; c - ToF-based displacement estimates for the movements with the amplitude of $4 \mathrm{~mm}$. 

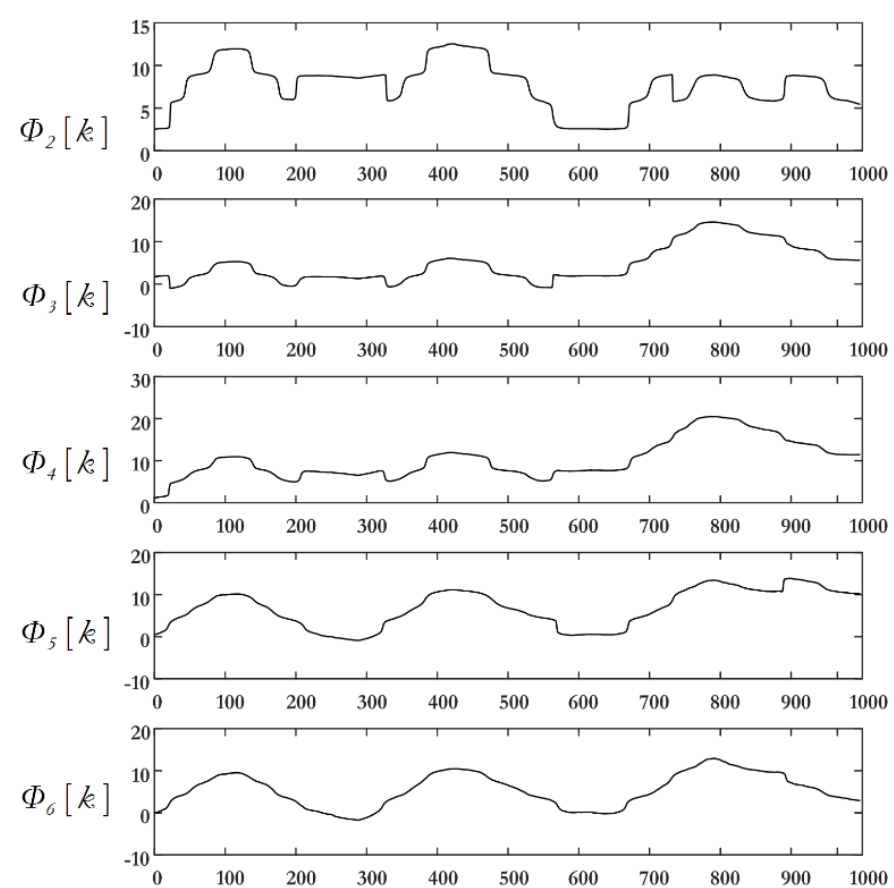

Figure 6. Absolute values of the phase spectrum components changing due to the human chest displacement during the respiration process.

Figure 5(a) contains ten graphs showing the absolute values of the phase spectrum components $\Phi_{m}[k]$ changing due to the aluminium sheet displacement with the amplitude of $50 \mu \mathrm{m}$. Figure 5(b) shows the absolute values of the phase spectrum component $\Phi_{m}[k]$ for the sheet's displacement with a $4 \mathrm{~mm}$ amplitude. Figure 5(c) shows the ToF-based displacement estimates for the movements with an amplitude of $4 \mathrm{~mm}$.

For the second and third experiments, the human chest was used as the object of interest. Figure 6 shows ten graphs demonstrating the absolute values of the phase spectrum components $\Phi_{m}[k]$, changing due to human chest
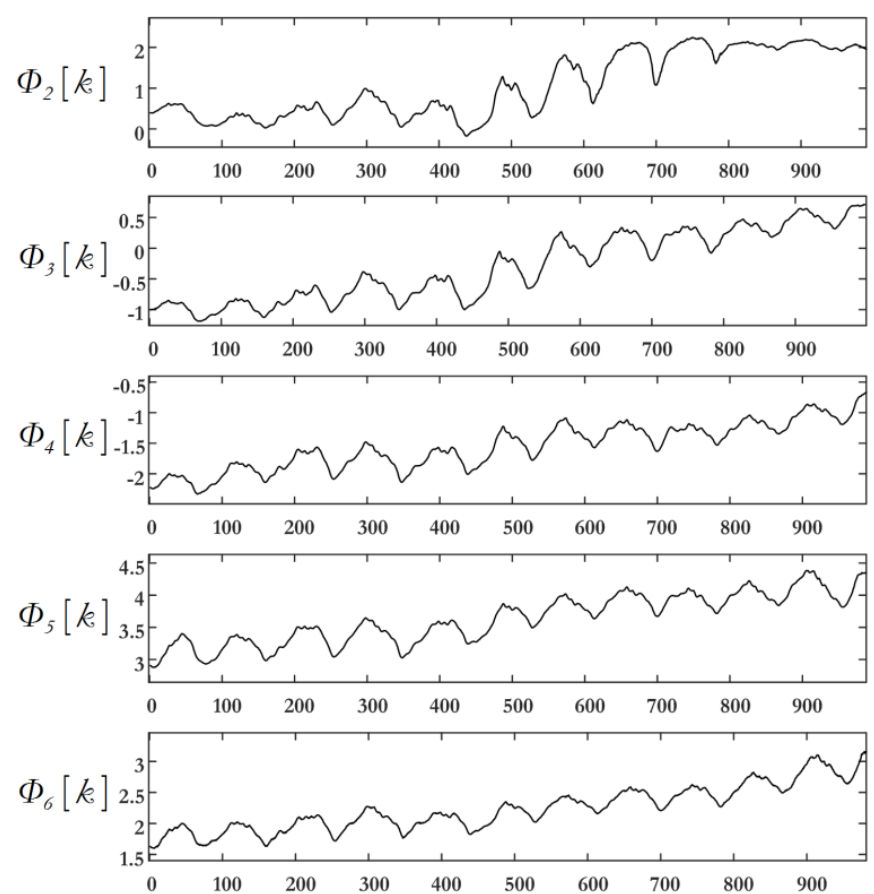

displacement during the respiration process, such values having been computed using the proposed method.

Figure 7 contains the ten graphs of absolute values of the phase spectrum components $\Phi_{m}[k]$, changing due to cardiac activity while a person was holding their breath.

\section{CONCLUSIONS}

It is possible to empirically select the relevant (i.e. those that are the most correlated with the object's movements) components of $\Phi_{m}[k]$ from the series computed by the proposed method. For the first experiment, these estimates are
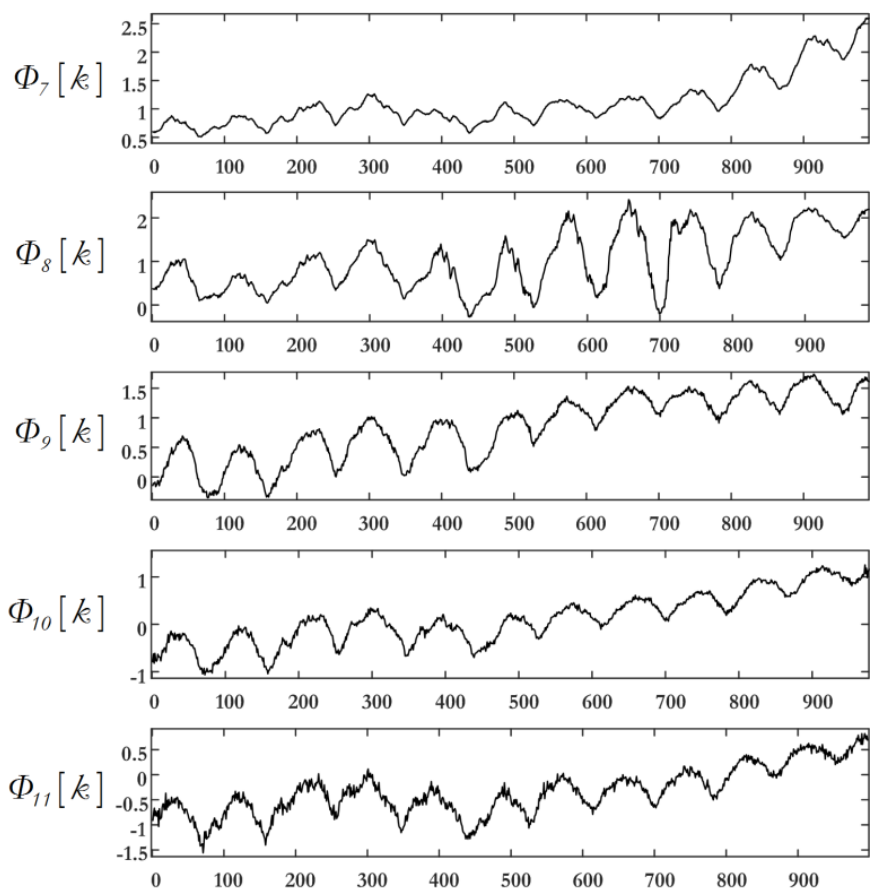

Figure 7. Absolute values of the phase spectrum components $\Phi_{m}[k]$ changing due to the human chest displacement caused by cardiac activity. 
under numbers 5, 6, 10; for the second experiment, these estimates are under numbers 7, 8, 9; and for the third experiment, these estimates are under numbers $5,6,8$.

Since the aluminium sheet used in the first experiment was moving with an amplitude of $50 \mu \mathrm{m}$, we can make a rough estimate of the proposed method's resolution, which is less than $50 \mu \mathrm{m}$ and, consequently, less than one hundredth of the ultrasonic wavelength with a frequency of $f_{u s}=40 \mathrm{kHz}$, making it sufficient for the purposes of respiration and heartbeat detection. However, the aforementioned estimate is highly conservative and will be a subject of future research.

According to the experimental results, the ToF method is inadequate for plotting the respiration and heartbeat graphs, due to its measurement resolution. It should be also noted that in all of the experiments, a considerable part of the target object was covered by the sensor's beam pattern, and the waveform of the echo signal's envelope depends highly upon object's shape. So, in the first experiment, all the surface points move equally linearly, but in the second and third experiments, the shape of the surface is subject to the nonlinear three-dimensional changes, causing sufficient changes in the waveform of the corresponding echo signal's envelope. For that reason, the ToF-based results contain more noise for the second and third experiments than for the first. Consequently, for most of the applications, the measurement resolution of this method is in close proximity to the wavelength.

Since the displacements caused by the respiration and heartbeat can be considered to be concentrated in the small neighbourhood of $d_{r e f}$, it is possible to provide accurate estimates based on the phase components. The proposed method is robust to such displacements, since the only thing that changes during the process is the set of the relevant phase spectrum components. The main source of such robustness is the fact that both the time at which the envelope exceeds the comparison threshold $u_{r e f}$ and all the information it contains is used to perform calculations. However, to survive the significant nonlinear changes in the object's surface over time, some combinations of the specific phase components should be used.

Therefore, the proposed method is suitable for small movement detection happening in any region of the sensor's beam pattern and can be applied by mobile robots to detect human's vital signs during a SAR operation.

An algorithm for the automatic selection of the most relevant phase spectrum components or combinations thereof based on a priori information about the target object and the measuring conditions poses another subject for a future research. Topics of interest also include the impact of the rough estimate $d_{r e f}$ on the relevant phase components selection and the compensation of the distortions caused by the air streams on the territory covered by the sensor's beam pattern.

\section{REFERENCES}

[1] E. M. Grigoryev, S. G. Gurzhin, V. I. Zhulev, M. B. Kaplan, V. G. Kryakov, E. M. Proshin, Technology and methods for formation of the complex magnetotherapy impact by the inductor array, Proc. of the $5^{\text {th }}$ Mediterranean Conference on Embedded Computing, MECO 2016, June 2016, Bar, Montenegro, pp. 419422.

[2] T. Bagosi, K. V. Hindriks, M. A. Neerincx, Ontological reasoning for human-robot teaming in search and rescue missions, Proc. of the $11^{\text {th }} \mathrm{ACM} / \mathrm{IEEE}$ International Conference on Human-Robot Interaction (HRI), 7-10 March 2016, Christchurch, New Zealand, pp. 595-596.

[3] F. Çakmak, E. Uslu, M. F. Amasyali, S. Yavuz, Hazmat charts detection on search and rescue league of RoboCup competitions, Proc. of the $25^{\text {th }}$ Signal Processing and Communications Applications Conference (SIU), 15-18 May 2017, Antalya, Turkey, pp. 1-4.

[4] D. J. Nallathambi, Comprehensive evaluation of the performance of rescue robots using victim robots, Proc. of the $4^{\text {th }}$ International Conference on Control, Automation and Robotics (ICCAR), 2023 April 2018, Auckland, New Zealand, pp. 60-64.

[5] R. Ventura, P. U. Lima, Search and rescue robots: the civil protection teams of the future, Proc. of the $3^{\text {rd }}$ International Conference on Emerging Security Technologies, 5-7 September 2012, Lisbon, Portugal, pp. 12-19.

[6] M. M. Saad, C. J. Bleakley, S. Dobson, Robust high-accuracy ultrasonic range measurement system, IEEE Trans. Instrum. Meas. 60(10) (2011) pp. 3334-3341.

[7] K. Yasuda, K. Takamura, T. Masuda, K. Magatani, A remote measurement method of the human biosignals, Proc. of the IEEE EMBS Asian-Pacific Conference on Biomedical Engineering, 2022 October 2003, Kyoto, Japan, pp. 288-289.

[8] C. Li, W. Chen, G. Liu, R. Yan, H. Xu, Y. Qi, A noncontact FMCW radar sensor for displacement measurement in structural health monitoring. Sensors 15(4) (2015) pp. 7412-7433.

[9] J. H. Lee, J. M. Hwang, D. H. Choi, S. O. Park, Noninvasive biosignal detection radar system using circular polarization. IEEE Transactions on Information Technology in Biomedicine 13(3) (2009) pp. 400-404.

[10] Pr. k Mane, K. N. Rao, Computational modelling of biosignalbased occupancy sensing system using Doppler radar, Proc. of the International Conference on Trends in Electronics and Informatics (ICEI), 11-12 May 2017, Tirunelveli, India, pp. 25-31.

[11] Texas Instruments Datasheet IWR1443 Single-Chip 76- to 81GHz mm Wave Sensor, SWRS211B - May 2017 - revised February 2018.

[12] J. C. Jackson, R. Summan, G. I. Dobie, S. M. Whiteley, S. G. Pierce, G. Hayward, Time-of-flight measurement techniques for airborne ultrasonic ranging. IEEE Transactions on Ultrasonics, Ferroelectrics, and Frequency Control 60(2) (2013) pp. 343-355.

[13] A. Saad, A. Radwan A, S. Sadek, S. Obeid, G. Zaharia, G. E. Zein, Remote monitoring of heart and respiration rate using a wireless microwave sensor, Proc. of the $12^{\text {th }}$ International Healthcare, Hospital Supplies and Medical Equipment Shows (Saudi Medicare 2009), May 2009, Riad, Saudi Arabia.

[14] S. Hirata, M. K. Kurosawa, T. Katagiri, Accuracy and resolution of ultrasonic distance measurement with high-time-resolution cross-correlation function obtained by single-bit signal processing. Acoust. Sci. \& Tech. 30(6) (2009) pp. 429-438.

[15] S. S. Huang, C. F. Huang, K. N. Huang, M. S. Young, A high accuracy ultrasonic distance measurement system using binary frequency shift-keyed signal and phase detection. The Review of Scientific Instruments 73(10) (2002) pp. 3671-3677.

[16] K. Y. Lee, C. F. Huang, S. S. Huang, K. N. Huang, M. S. Young, A high-resolution ultrasonic distance measurement system using Vernier caliper phase meter. IEEE Transactions on Instrumentation and Measurement 61(11) (2012) pp. 2924-2931.

[17] V. Agarwal, N. V. Murali, C. Chandramouli, A cost-effective ultrasonic sensor-based driver-assistance system for congested traffic conditions, IEEE Transactions on Intelligent Transportation Systems 10(3) (2009) pp. 486-498.

[18] Y. P. Huang, J. S. Wang, K. N. Huang, C. T. Ho, J. D. Huang, M. S. Young, Envelope pulsed ultrasonic distance measurement system based upon amplitude modulation and phase modulation. The Review of Scientific Instruments 78(6) (2007) p. 06510. 